DOI: 10.21915 /BIMAS.2019102

\title{
ON FUSION PROCEDURE FOR THE TWO-PARAMETER QUANTUM ALGEBRA IN TYPE $A$
}

\author{
NAIHUAN JING ${ }^{1, a}$ AND MING LIU ${ }^{2, b}$ \\ Dedicated to Robert Griess, Jr. in honor of his $71^{\text {st }}$ birthday \\ ${ }^{1}$ Department of Mathematics, North Carolina State University, Raleigh, NC 27695, USA. \\ ${ }^{a}$ E-mail: jing@math.ncsu.edu \\ ${ }^{2}$ School of Mathematics, South China University of Technology, Guangzhou 510640, China. \\ ${ }^{b}$ E-mail: mamliu@scut.edu.cn
}

\begin{abstract}
Finite dimensional irreducible modules of the two-parameter quantum enveloping algebra $U_{r, s}\left(\mathfrak{s l}_{n}\right)$ are explicitly constructed using the fusion procedure when $r s^{-1}$ is generic. This provides an alternative and combinatorial description of the Schur-Weyl duality for the two-parameter quantum linear algebras of type $A$.
\end{abstract}

\section{Introduction}

Schur-Weyl duality is one of the main methods to construct irreducible modules of the classical simple Lie groups out of the fundamental representations [22]. The quantum version for the quantum enveloping algebra $U_{q}\left(\mathfrak{s l}_{n}\right)$ and the Hecke algebra $H_{q}\left(\mathfrak{S}_{m}\right)$ has played an important role in the fervent development of quantum groups. They provide one of the first examples [13] to show the similarity between the classical and quantum theories.

Two-parameter general and special linear quantum groups [21, 8, 4] are further generalization of the corresponding one-parameter Drinfeld-Jimbo quantum groups $[7,12]$. The two-parameter quantum groups also had their origin in the quantum inverse scattering method [20] as well as other approaches $[14,6]$. In particular, the Schur-Weyl duality was also generalized

Received February 7, 2017.

AMS Subject Classification: Primary: 17B30; Secondary: 17B68.

Key words and phrases: Two-parameter quantum groups, fusion procedure, Schur-Weyl duality. 
to the two-parameter case [2]. As in the classical situation, let $V$ be the natural $n$-dimensional module of $U_{r, s}\left(\mathfrak{s l}_{n}\right)$, then arbitrary irreducible modules can be constructed using the $(r, s)$-symmetric tensor $S_{r, s}^{2}(V)$ and the R-matrix $R=R_{V V}$ for two-parameter quantum group $U_{r, s}\left(\mathfrak{s l}_{n}\right)$. Alternatively any finite dimensional irreducible $U_{r, s}\left(\mathfrak{s l}_{n}\right)$-module can be built from the tensor product $V^{\otimes m}$ using the symmetry of the Hecke algebra $H_{q}\left(\mathfrak{S}_{m}\right)$, where $q=\sqrt{s / r}$.

In this work we give an alternative description of all irreducible representations ( $r s^{-1}$ is not a root of unity) using the developments [11] of the fusion procedure [3] in the quantum inverse scattering method. It seems that the two-parameter case can be treated quite similarly using the fusion procedure, thus one can more or less apply the known results from the one-parameter case to get corresponding formulas. As there is an abstract argument available to construct the irreducible modules in the two-parameter case, we nevertheless give a detailed description of all irreducible $U_{r, s}\left(\mathfrak{s l}_{n}\right)$-modules using the fusion procedure to supplement the existing theory.

Much of the results in the paper are expected for the experts, and we hope the current presentation can further show the similarity and connection to the one-parameter case. The one-parameter case is adapted into the two parameter situation in a self-contained manner and we try to be complete as much as possible for pedagogical purpose.

\section{Two-parameter Quantum Group $U_{r, s}\left(\mathfrak{s l}_{n}\right)$ and R-matrix}

We start with the basic definition of the two-parameter quantum groups $U_{r, s}\left(\mathfrak{g l}_{n}\right), U_{r, s}\left(\mathfrak{s l}_{n}\right)$ following the notations in [2]. Let $\Pi=\left\{\alpha_{j}=\epsilon_{j}-\epsilon_{j+1} \mid j=\right.$ $1,2, \ldots, n-1\}$ be the set of the simple roots of type $A_{n-1}$, where $\epsilon_{1}, \epsilon_{2}, \ldots, \epsilon_{n}$ are an orthonormal basis of a Euclidean space with inner product $\langle$,$\rangle . The$ root system is then $\Phi=\left\{\epsilon_{i}-\epsilon_{j} \mid 1 \leq i \neq j \leq n\right\}$.

Fix two distinct nonzero complex numbers $r, s$, and assume they are in general position.

Definition 2.1. The two-parameter quantum enveloping algebra $\widetilde{U}=U_{r, s}\left(\mathfrak{g l}_{n}\right)$ is the unital associative algebra over $\mathbb{C}$ generated by $e_{j}, f_{j}$, $(1 \leq j<n)$ and $a_{i}^{ \pm 1}, b_{i}^{ \pm 1},(1 \leq i \leq n)$ with the following relations:

(R1) $a_{i}^{ \pm 1}, b_{i}^{ \pm 1}$ commute with each other, and $a_{i} a_{i}^{-1}=b_{i} b_{i}^{-1}=1$, 
(R2) $a_{i} e_{j} a_{i}^{-1}=r^{\left\langle\epsilon_{i}, \alpha_{j}\right\rangle} e_{j}$ and $a_{i} f_{j} a_{i}^{-1}=r^{-\left\langle\epsilon_{i}, \alpha_{j}\right\rangle} f_{j}$,

(R3) $b_{i} e_{j} b_{i}^{-1}=s^{\left\langle\epsilon_{i}, \alpha_{j}\right\rangle} e_{j}$ and $b_{i} f_{j} b_{i}^{-1}=s^{-\left\langle\epsilon_{i}, \alpha_{j}\right\rangle} f_{j}$,

(R4) $\left[e_{i}, f_{j}\right]=\frac{\delta_{i j}}{r-s}\left(a_{i} b_{i+1}-a_{i+1} b_{i}\right)$,

(R5) $\left[e_{i}, e_{j}\right]=\left[f_{i}, f_{j}\right]=0$, if $|i-j|>1$,

(R6) $e_{i}^{2} e_{i+1}-(r+s) e_{i} e_{i+1} e_{i}+r s e_{i+1} e_{i}^{2}=0$ and

$e_{i} e_{i+1}^{2}-(r+s) e_{i+1} e_{i} e_{i+1}+r s e_{i+1}^{2} e_{i}=0$,

(R7) $f_{i}^{2} f_{i+1}-\left(r^{-1}+s^{-1}\right) f_{i} f_{i+1} f_{i}+r^{-1} s^{-1} f_{i+1} f_{i}^{2}=0$ and

$f_{i} f_{i+1}^{2}-\left(r^{-1}+s^{-1}\right) f_{i+1} f_{i} f_{i+1}+r^{-1} s^{-1} f_{i+1}^{2} f_{i}=0$,

where $[x, y]=x y-y x$ is the commutator.

The algebra $U=U_{r, s}\left(\mathfrak{s l}_{n}\right)$ is the subalgebra of $\widetilde{U}=U_{r, s}\left(\mathfrak{g l}_{n}\right)$ generated by the elements $e_{j}, f_{j}, \omega_{j}$ and $\omega_{j}^{\prime}(1 \leq j<n)$, where $\omega_{j}=a_{j} b_{j+1}$, and $\omega_{j}^{\prime}=a_{j+1} b_{j}$. These elements satisfy the relations (R5)-(R7) along with

$\left(\mathrm{R} 1^{\prime}\right)$ The $\omega_{i}, \omega_{j}^{\prime}$ are invertible and they all commute with each other,

$\left(\mathrm{R} 2^{\prime}\right) \omega_{i} e_{j}=r^{\left\langle\epsilon_{i}, \alpha_{j}\right\rangle} s^{\left\langle\epsilon_{i+1}, \alpha_{j}\right\rangle} e_{j} \omega_{i}$ and $\omega_{i} f_{j}=r^{-\left\langle\epsilon_{i}, \alpha_{j}\right\rangle} s^{-\left\langle\epsilon_{i+1}, \alpha_{j}\right\rangle} f_{j} \omega_{i}$,

$\left(\mathrm{R}^{\prime}\right) \omega_{i}^{\prime} e_{j}=r^{\left\langle\epsilon_{i+1}, \alpha_{j}\right\rangle} s^{\left\langle\epsilon_{i}, \alpha_{j}\right\rangle} e_{j} \omega_{i}^{\prime}$ and $\omega_{i}^{\prime} f_{j}=r^{-\left\langle\epsilon_{i+1}, \alpha_{j}\right\rangle} s^{-\left\langle\epsilon_{i}, \alpha_{j}\right\rangle} f_{j} \omega_{i}^{\prime}$,

$\left(\mathrm{R} 4^{\prime}\right)\left[e_{i}, f_{j}\right]=\frac{\delta_{i j}}{r-s}\left(\omega_{i}-\omega_{i}^{\prime}\right)$.

Clearly when $r=q, s=q^{-1}$, the algebra $U$ modulo the ideal generated by the elements $\omega_{j}^{-1}-\omega_{j}^{\prime}, 1 \leq j<n$, is isomorphic to $U_{q}\left(\mathfrak{s l}_{n}\right)$.

The algebra $U_{r, s}\left(\mathfrak{s l}_{n}\right)$ is a Hopf algebra under the comultiplication $\Delta$ such that $\omega_{i}, \omega_{i}^{\prime}$ are group-like elements and the other nontrivial comultiplications, counits and antipodes are given by:

$$
\begin{aligned}
\Delta\left(e_{i}\right) & =e_{i} \otimes 1+\omega_{i} \otimes e_{i}, \Delta\left(f_{i}\right)=1 \otimes f_{i}+f_{i} \otimes \omega_{i}^{\prime} \\
\epsilon\left(e_{i}\right) & =\epsilon\left(f_{i}\right)=0, S\left(e_{i}\right)=-\omega_{i}^{-1} e_{i}, S\left(f_{i}\right)=-f_{i} \omega_{i}^{\prime-1}
\end{aligned}
$$

The representation theory of $U_{r, s}\left(\mathfrak{s l}_{n}\right)$ is quite similar to that of oneparameter case. We recall some of the basic notations for later discussion. Let $\Lambda=\mathbb{Z} \epsilon_{1} \oplus \mathbb{Z} \epsilon_{2} \oplus \cdots \oplus \mathbb{Z} \epsilon_{n}$ be the weight lattice of $\mathfrak{g l}_{n}, Q=\mathbb{Z} \Phi$ the root lattice, and $Q_{+}=\sum_{i=1}^{n-1} \mathbb{Z}_{\geq 0} \alpha_{i}$, where $\epsilon_{i}$ are the orthonomal vectors as before. Recall that $\Lambda$ is equipped with the partial order given by $\nu \leq \lambda$ if and only if $\lambda-\nu \in Q_{+}$. 
For each $\lambda \in \Lambda$ one defines the algebra homomorphism $\widehat{\lambda}: \widetilde{U}^{0} \rightarrow \mathbb{C}$ by:

$$
\widehat{\lambda}\left(a_{i}\right)=r^{\left\langle\epsilon_{i}, \lambda\right\rangle}, \quad \widehat{\lambda}\left(b_{i}\right)=s^{\left\langle\epsilon_{i}, \lambda\right\rangle},
$$

where $\widetilde{U}^{0}$ is the subalgebra of $U_{r, s}\left(\mathfrak{g l}_{n}\right)$ generated by $a_{i}^{ \pm 1}, b_{i}^{ \pm 1}(1 \leq i \leq n)$. Then the restriction of $\widehat{\lambda}: U^{0} \rightarrow \mathbb{C}$ of $\widehat{\lambda}$ to the subalgebra $U^{0}$ of $U$ generated by $\omega_{j}, \omega_{j}^{\prime}(1 \leq j<n)$ satisfies:

$$
\widehat{\lambda}\left(\omega_{j}\right)=r^{\left\langle\epsilon_{j}, \lambda\right\rangle} s^{\left\langle\epsilon_{j+1}, \lambda\right\rangle}, \quad \widehat{\lambda}\left(\omega_{j}^{\prime}\right)=r^{\left\langle\epsilon_{j+1}, \lambda\right\rangle} s^{\left\langle\epsilon_{i}, \lambda\right\rangle} .
$$

It was shown in [1] when $r s^{-1}$ is not a root of unity, the homomorphisms $\widehat{\lambda}=\widehat{\mu}$ if and only if the corresponding weights $\lambda=\mu$. These homomorphisms are called generalized weights. For an algebra homomorphism $\chi: U^{0} \mapsto \mathbb{C}$ one defines the generalized weight subspace of $U_{r, s}$-module $\mathrm{M}$ by

$M_{\chi}=\left\{v \in M \mid\left(\omega_{i}-\chi\left(\omega_{i}\right)\right)^{m} v=\left(\omega_{i}^{\prime}-\chi\left(\omega_{i}^{\prime}\right)\right)^{m} v=0\right.$, for all $i$ and for some $\left.m\right\}$.

If $m=1$, the subspace $M_{\chi}$ becomes a weight subspace associated with the homomorphism $\chi$. Since $U^{0}$ is commutative, it is easy to see that any finite dimensional $U_{r, s}$-module $M$ can be decomposed into a sum of generalized weight subspaces:

$$
M=\bigoplus_{\chi} M_{\chi}
$$

When all generalized weights in $M$ are of the form $\chi \cdot(-\widehat{\alpha})$ for a fixed $\chi$ and $\alpha$ varying in $Q_{+}$(here $\chi \cdot(-\widehat{\alpha})\left(\omega_{i}\right)=\chi\left(\omega_{i}\right)(-\widehat{\alpha})\left(\omega_{i}\right)$ and $\chi \cdot(-\widehat{\alpha})\left(\omega_{i}^{\prime}\right)=$ $\left.\chi\left(\omega_{i}^{\prime}\right)(-\widehat{\alpha})\left(\omega_{i}^{\prime}\right)\right)$, we say $M$ is a highest weight module of weight $\chi$ and write $M=M(\chi)$. Benkart and Witherspoon [2] have shown that when $M$ is simple, all generalized weight subspaces are actually weight subspaces. Moreover, if all generalized weights are of the form $\hat{\lambda}$ for $\lambda \in \Lambda$, we will simply write $M_{\lambda}$ for $M_{\widehat{\lambda}}$, and similarly the highest weight module $M(\widehat{\lambda})$ will be denoted as $M(\lambda)$.

One can also define the notion of Verma modules $M(\lambda)$ as in the classical situation. It is known [2] that all finite dimensional simple $U_{r, s}\left(\mathfrak{s l}_{n}\right)$-modules are realized as simple quotients of Verma modules. We will write by $V(\lambda)$ the simple quotient of the Verma module $M(\lambda)$. 
Now let us look at the simplest irreducible module $V\left(\bar{\omega}_{1}\right)$. Let $V$ be the $n$-dimensional vector space over $\mathbb{C}$ with basis $\left\{v_{j} \mid 1 \leq j \leq n\right\}$, and $E_{i j} \in \operatorname{End}(V)$ be defined by $E_{i j} v_{k}=\delta_{j k} v_{i}$. Define the $U_{r, s}\left(\mathfrak{s l}_{n}\right)$-action on $V$ by

$$
\begin{aligned}
& e_{j}=E_{j, j+1}, \quad f_{j}=E_{j+1, j}, \\
& \omega_{j}=r E_{j j}+s E_{j+1, j+1}+\sum_{k \neq j, j+1} E_{k k}, \\
& \omega_{j}^{\prime}=s E_{j j}+r E_{j+1, j+1}+\sum_{k \neq j, j+1} E_{k k},
\end{aligned}
$$

where $1 \leq j \leq n-1$. It is clear that $V=\bigoplus_{j=1}^{n} V_{\epsilon_{j}}$ and it is the simple $U_{r, s}\left(\mathfrak{s l}_{n}\right)$-module $V\left(\bar{\omega}_{1}\right)$.

Let $\check{R}=\check{R}_{V V}: V \otimes V \longrightarrow V \otimes V$ be the $R$-matrix defined by

$$
\check{R}=\sum_{i=1}^{n} E_{i i} \otimes E_{i i}+r \sum_{i<j} E_{j i} \otimes E_{i j}+s^{-1} \sum_{i<j} E_{i j} \otimes E_{j i}+\left(1-r s^{-1}\right) \sum_{i<j} E_{j j} \otimes E_{i i},
$$

which is essentially determined by the simple module $V$ and the comultiplication $\Delta$ (see Prop. 2.2) . For each $1 \leq i<k$ let $\check{R}_{i}$ be the isomorphism on $V^{\otimes k}$ defined by

$$
\breve{R}_{i}\left(w_{1} \otimes w_{2} \otimes \cdots \otimes w_{k}\right)=w_{1} \otimes \cdots \otimes \check{R}\left(w_{i} \otimes w_{i+1}\right) \otimes w_{i+2} \otimes \cdots \otimes w_{k}
$$

Then we have the braid relations:

$$
\check{R}_{i} \check{R}_{i+1} \check{R}_{i}=\check{R}_{i+1} \check{R}_{i} \check{R}_{i+1} \quad \text { for } \quad 1 \leq i<k-1
$$

The construction also implies that for $|i-j| \geq 2$,

$$
\check{R}_{i} \check{R}_{j}=\check{R}_{j} \check{R}_{i}
$$

Moreover one can directly check that

$$
\check{R}_{i}^{2}=\left(1-\frac{r}{s}\right) \check{R}_{i}+\frac{r}{s} I d
$$

for all $1 \leq i<k$. In particular, the minimum polynomial of $\check{R}$ on $V \otimes V$ is $(t-1)\left(t+\frac{r}{s}\right)$ if $s \neq-r[2]$. 
Proposition $2.2([2])$. The endomorphisms $\check{R}_{i} \in \operatorname{End}\left(V^{\otimes k}\right)$ commute with the action of $U_{r, s}\left(\mathfrak{s l}_{n}\right)$ on $V^{\otimes k}$.

Proof. By the Hopf algebra structure of $U_{r, s}\left(\mathfrak{s l}_{n}\right)$ it is enough to check this for $k=2$. Then it is a direct verification to confirm that $\check{R}$ commutes with $\Delta\left(e_{i}\right), \Delta\left(f_{i}\right), \Delta\left(\omega_{i}\right), \Delta\left(\omega_{i}^{\prime}\right)$ for $i=1, \cdots, n-1$ on the fundamental representation $V\left(\bar{\omega}_{1}\right)$.

\section{Yang-Baxterization and the Wedge Modules of $U_{r, s}\left(\mathfrak{s l}_{n}\right)$}

The fusion procedure relies on the spectral parameter dependent $\mathrm{R}$ matrix $\check{R}(z)$, which satisfies the so-called quantum Yang-Baxter equation. One form of the Yang-Baxter equation (YBE) is the following matrix equation on $V^{\otimes 3}$ :

$$
\check{R}_{1}(z) \check{R}_{2}(z w) \check{R}_{1}(w)=\check{R}_{2}(w) \check{R}_{1}(z w) \check{R}_{2}(z)
$$

where $\check{R}(0)=\check{R}$. The Yang-Baxterization method recovers the spectral parameter dependent R-matrix $\check{R}(z)$ from the initial condition (2.5) at $z=0$.

The Yang-Baxterization process was carried out for the two-parameter $R$-matrix in [16] using the method of [9].

Proposition 3.1 ([16]). For the braid group representation $\breve{R}=\check{R}_{V V}$, the $R$-matrix $\check{R}(z)$ is given by

$$
\begin{aligned}
\check{R}(z)= & \left(1-z r s^{-1}\right) \sum_{i=1}^{n} E_{i i} \otimes E_{i i}+(1-z)\left(r \sum_{i>j}+s^{-1} \sum_{i<j}\right) E_{i j} \otimes E_{j i} \\
& +z\left(1-r s^{-1}\right) \sum_{i<j} E_{i i} \otimes E_{j j}+\left(1-r s^{-1}\right) \sum_{i>j} E_{i i} \otimes E_{j j} .
\end{aligned}
$$

Remark 3.2. Clearly $\check{R}(0)=\check{R}$. Moreover, when $r=q$ and $s=q^{-1}$, the R-matrix $\check{R}(z)$ turns into

$$
\begin{aligned}
\check{R}_{q}(z)= & \left(1-z q^{2}\right) \sum_{i=1}^{n} E_{i i} \otimes E_{i i}+(1-z) q \sum_{i \neq j} E_{i j} \otimes E_{j i} \\
& +\left(1-q^{2}\right)\left(\sum_{i>j}+z \sum_{i<j}\right) E_{i i} \otimes E_{j j}
\end{aligned}
$$


which is exactly the Jimbo R-matrix for the quantum affine algebra $U_{q}\left(\widehat{\mathfrak{s l}}_{n}\right)$ [12]. In this regard we can view $\check{R}(z)$ as an $(r, s)$-analogue of the R-matrix $\check{R}_{q}(z)$ of the quantum affine algebra $U_{q}\left(\widehat{\mathfrak{s l}}_{n}\right)$ [10]. The one-parameter $R$ matrix can also be used to treat the quantum algebra $U_{q}(\mathfrak{g l}(m \mid n))$ [19].

For convenience, we introduce a normalized R-matrix with two spectral parameters

$$
\begin{aligned}
\check{R}(x, y)= & \frac{s y \check{R}(x / y)}{y-x} \\
= & \frac{s y-r x}{y-x} \sum_{i=1}^{n} E_{i i} \otimes E_{i i}+\left(s r \sum_{i>j}+\sum_{i<j}\right) E_{i j} \otimes E_{j i} \\
& +\frac{(s-r) x}{y-x} \sum_{i<j} E_{i i} \otimes E_{j j}+\frac{(s-r) y}{y-x} \sum_{i>j} E_{i i} \otimes E_{j j} .
\end{aligned}
$$

The original YBE immediately implies the following Yang-Baxter equation:

$$
\check{R}_{i}(x, y) \check{R}_{i+1}(x, z) \check{R}_{i}(y, z)=\check{R}_{i+1}(y, z) \check{R}_{i}(x, z) \check{R}_{i+1}(x, y)
$$

Let $S_{r, s}^{2}(V)$ be the subspace of $V \otimes V$ spanned by $\left\{v_{i} \otimes v_{i} \mid 1 \leq i \leq\right.$ $n\} \cup\left\{v_{i} \otimes v_{j}+s v_{j} \otimes v_{i} \mid 1 \leq i<j \leq n\right\}$ and $\Lambda_{r, s}^{2}(V)$ be the subspace of $V \otimes V$ spanned by $\left\{v_{i} \otimes v_{j}-r v_{j} \otimes v_{i} \mid 1 \leq i<j \leq n\right\}$.

Proposition 3.3 ([16]). The subspace $S_{r, s}^{2}(V)$ is equal to the image of $\check{R}\left(1, r^{-1} s\right)$ on $V \otimes V$, and $\Lambda_{r, s}^{2}(V)$ is equal to the image of $\check{R}\left(1, r s^{-1}\right)$ on $V \otimes V$.

Remark 3.4. The above result is equivalent to $S_{r, s}^{2}(V)=\operatorname{Ker} \check{R}\left(1, s r^{-1}\right)$ and $\Lambda_{r, s}^{2}(V)=\operatorname{Ker} \check{R}\left(1, s^{-1} r\right)$. This suggests that special values of the YangBaxter matrix can lead to irreducible modules, which we will show in general in Section 6.

Proposition 3.5 ([16]). The kth fundamental representation of $U_{r, s}\left(\mathfrak{s l}_{n}\right)$ can be realized as the following quotient of the $k$-fold tensor product

$$
V^{\otimes k} / \sum_{i=0}^{k-2} V^{\otimes i} \otimes S_{r, s}^{2}(V) \otimes V^{\otimes(k-i-2)} \cong V\left(\bar{\omega}_{k}\right) .
$$


In Section 6 we will give an alternative way to construct all irreducible modules using the Yang-Baxter equation.

\section{The Hecke Algebra and the Schur-Weyl Duality for $U_{r, s}\left(\mathfrak{g l}_{n}\right)$}

For any $r, s \in \mathbb{C} \backslash\{0\}$, we introduce the Hecke algebra $H_{m}(r, s)$ as follows.

Definition 4.1. The Hecke algebra $H_{m}(r, s)$ is the unital associative algebra over $\mathbb{C}$ with generators $T_{i}, 1 \leq i<m$, subject to the following relations:

(H1): $T_{i} T_{i+1} T_{i}=T_{i+1} T_{i} T_{i+1}, \quad 1 \leq i<m-1$,

(H2): $T_{i} T_{j}=T_{j} T_{i}, \quad|i-j|>1$,

(H3): $\left(T_{i}-1\right)\left(T_{i}+\frac{r}{s}\right)=0$.

Remark 4.2. When $r \neq 0$, the elements $t_{i}=\sqrt{\frac{s}{r}} T_{i}$ satisfy

$$
\left(\mathrm{H} 3^{\prime}\right):\left(t_{i}-\sqrt{\frac{s}{r}}\right)\left(t_{i}+\sqrt{\frac{r}{s}}\right)=0 .
$$

If we set $q=\sqrt{\frac{s}{r}}$, the two parameter Hecke algebra $H_{m}(r, s)$ is isomorphic to $H_{m}(q)$, the Hecke algebra associated to the symmetric group $\mathfrak{S}_{m}$. By a well-known result of Hecke algebras $H_{m}(r, s)$ is semisimple whenever $\sqrt{\frac{s}{r}}$ is not a root of unity.

From Section 2, it is easy to verify that the $U_{r, s}\left(\mathfrak{g l}_{n}\right)$-module $V^{\otimes m}$ affords a representation of Hecke algebra $H_{m}(r, s)$ :

$$
\begin{aligned}
& H_{m}(r, s) \rightarrow \operatorname{End}_{U_{r, s}\left(\mathfrak{g l}_{n}\right)}\left(V^{\otimes m}\right) \\
& T_{i} \mapsto \check{R}_{i} \quad(1 \leq i<m) .
\end{aligned}
$$

Benkart and Witherspoon [2] gave a two parameter analogue of the Schur-Weyl duality for $U_{r, s}\left(\mathfrak{s l}_{n}\right)$ and the Hecke algebra $H_{r, s}\left(\mathfrak{S}_{k}\right)$ associated with the symmetric group $\mathfrak{S}_{k}$, which we recall as follows.

Proposition $4.3([2])$. Assume $r s^{-1}$ is not a root of unity. Then:

(i) $H_{m}(r, s)$ maps surjectively onto $\operatorname{End}_{U_{r, s}\left(\mathfrak{g l}_{n}\right)}\left(V^{\otimes m}\right)$.

(ii) if $n \geq m, H_{m}(r, s)$ is isomorphic to $\operatorname{End}_{U_{r, s}\left(\mathfrak{g l}_{n}\right)}\left(V^{\otimes m}\right)$. 
Let $\lambda=\left(\lambda_{1}, \lambda_{2}, \ldots, \lambda_{l}\right)$ be a partition, where $l=l(\lambda)$ is the length.

Corollary 4.4. When $r^{-1}$ is not a root of unity, as an $\left(U_{r, s}\left(\mathfrak{g l}_{n}\right) \otimes H_{m}(r, s)\right)$ -module, the space $V^{\otimes m}$ has the following decomposition:

$$
V^{\otimes m} \cong \bigoplus_{\lambda} V_{\lambda} \otimes V^{\lambda}
$$

where the partition $\lambda$ of $m$ runs over the set of partition such that $l(\lambda) \leq n$, $V_{\lambda}$ is the $U_{r, s}\left(\mathfrak{g l}_{n}\right)$-module associated to $\lambda$, and $V^{\lambda}$ is the $H_{m}(r, s)$-module corresponding to $\lambda$.

Remark 4.5. The above theorem is a two-parameter version of the wellknown result of Jimbo [13].

In section 6 we will give detailed information on the idempotents, which will then give a realization of all irreducible modules.

\section{The Orthogonal Primitive Idempotents of $H_{m}(r, s)$}

For any index $i=1, \ldots, m-1$, let $s_{i}=(i, i+1)$ be the adjacent transposition in the symmetric group $\mathfrak{S}_{m}$. Take any element $\sigma \in \mathfrak{S}_{m}$ and choose a reduced decomposition $\sigma=s_{i_{1}} s_{i_{2}} \cdots s_{i_{k}}$. Denote $T_{\sigma}=T_{i_{1}} T_{i_{2}} \cdots T_{i_{k}}$, this element in $H_{m}(r, s)$ does not depend on the reduced decompositions of $\sigma[11]$.

The Jucys-Murphy elements of $H_{m}(r, s)$ are defined inductively by

$$
y_{1}=1, \quad y_{k+1}=\frac{s}{r} T_{k} y_{k} T_{k},
$$

where $k=1, \ldots, m-1$. These elements satisfy

$$
y_{k} T_{l}=T_{l} y_{k}, \quad l \neq k, k-1
$$

In particular, $y_{1}, y_{2}, \ldots, y_{m}$ generate a commutative subalgebra of $H_{m}(r, s)$. For any $k=1, \ldots, m$, we let $w_{k}$ denote the unique longest element of symmetric group $\mathfrak{S}_{k}$, which is regarded as a natural subgroup of $\mathfrak{S}_{m}$. The corresponding elements $T_{w_{k}} \in H_{m}(r, s)$ are given by $T_{\omega_{1}}=1$ and

$$
T_{w_{k}}=T_{1}\left(T_{2} T_{1}\right) \cdots\left(T_{k-2} T_{k-3} \cdots T_{1}\right)\left(T_{k-1} T_{k-2} \cdots T_{1}\right)
$$




$$
=\left(T_{1} \cdots T_{k-2} T_{k-1}\right)\left(T_{1} \cdots T_{k-3} T_{k-2}\right) \cdots\left(T_{1} T_{2}\right) T_{1}, k=2, \ldots, m .
$$

It can be verified easily that

$$
\begin{array}{rlrl}
T_{w_{k}} T_{j} & =T_{k-j} T_{w_{k}} & 1 \leq j<k \leq m \\
T_{w_{k}}^{2}=\left(\frac{r}{s}\right)^{\frac{k(k-1)}{2}} y_{1} y_{2} \cdots y_{k}, & k=1, \ldots, m .
\end{array}
$$

For each $i=1, \ldots, m-1$, we define the elements [18]:

$$
T_{i}(x, y)=s T_{i}+\frac{s-r}{\frac{y}{x}-1},
$$

where $x, y$ are complex variables. We will regard $T_{i}(x, y)$ as a rational functions in $x, y$ with values in $H_{m}(r, s)$. These functions satisfy the braid relations:

$$
T_{i}(x, y) T_{i+1}(x, z) T_{i}(y, z)=T_{i+1}(y, z) T_{i}(x, z) T_{i+1}(x, y)
$$

We will identify a partition $\lambda=\left(\lambda_{1}, \lambda_{2}, \ldots, \lambda_{l}\right)$ of $m$ with its Young diagram. The Young diagram is a left-justified array of rows of cells such that the first row contains $\lambda_{1}$ cells, the second row contains $\lambda_{2}$ cells, etc. A cell outside $\lambda$ is called addable to $\lambda$ if the union of $\lambda$ and the cell is a (proper) Young diagram. A $\lambda$-tableau is obtained by filling in the cells of the Young diagram bijectively with the numbers $1, \ldots, m$. A tableau $T$ is called standard if the entries of the tableau increase along the rows and down the columns. If a cell occurs in the $(\mathrm{i}, \mathrm{j})$-th position, its $(\mathrm{r}, \mathrm{s})$-content will be defined as $\left(\frac{s}{r}\right)^{j-i}$. Let $\sigma_{k}$ denote the $(\mathrm{r}, \mathrm{s})$-content of the cell occupied by $k$ in $T$.

Example 5.1. For $\lambda=(2,1)$, the corresponding Young diagram is

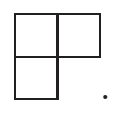

The cells at the positions $(1,3),(2,2),(3,1)$ are the addable cells. For the $\lambda$-tableau $T=\frac{13}{2}$, its $(r, s)$-contents are $\sigma_{1}=1, \sigma_{2}=\frac{r}{s}, \sigma_{3}=\frac{s}{r}$.

A set of primitive idempotents of $H_{m}(r, s)$ parameterized by partitions $\lambda$ of $m$ and the standard $\lambda$-tableaux $T$ can now be defined inductively by 
the following rule [5]. Set $E_{T}^{\lambda}=1$ if $m=1$, whereas for $m \geq 2$, one defines inductively

$$
E_{T}^{\lambda}=E_{U}^{\mu} \frac{\left(y_{m}-\rho_{1}\right) \cdots\left(y_{m}-\rho_{k}\right)}{\left(\sigma-\rho_{1}\right) \cdots\left(\sigma-\rho_{k}\right)},
$$

where $U$ is the tableau obtained from $T$ by removing the cell $\alpha$ occupied by $m, \mu$ is the shape of $U$, and $\rho_{1}, \ldots, \rho_{k}$ are the $(\mathrm{r}, \mathrm{s})$-contents of all the addable cells of $\mu$ except for $\alpha$, while $\sigma$ is the $(\mathrm{r}, \mathrm{s})$-content of the latter.

These elements satisfy the characteristic property that if $\lambda$ and $\lambda^{\prime}$ are partitions of $m$

$$
E_{T}^{\lambda} E_{T^{\prime}}^{\lambda^{\prime}}=\delta_{\lambda, \lambda^{\prime}} \delta_{T, T^{\prime}} E_{T}^{\lambda}
$$

for arbitrary standard tableaux $T$ and $T^{\prime}$ of shapes $\lambda$ and $\lambda^{\prime}$ respectively. Moreover,

$$
\sum_{\lambda} \sum_{T} E_{T}^{\lambda}=1
$$

summed over all partitions $\lambda$ of $m$ and all the standard $\lambda$-tableaux $T$.

\section{Fusion Formulas for the Orthogonal Primitive Idempotents of$$
H_{m}(r, s)
$$

We now apply the fusion formulas [11] to the situation of the twoparameter quantum algebra to derive a corresponding formula for the idempotents of $H_{m}(r, s)$, which can then be used to construct all the irreducible $U_{r, s}\left(\mathfrak{s l}_{n}\right)$-modules.

Let $\lambda=\left(\lambda_{1}, \ldots, \lambda_{l}\right)$ be a partition of $m$, the conjugate partition $\lambda^{\prime}=$ $\left(\lambda_{1}^{\prime}, \ldots, \lambda_{l^{\prime}}^{\prime}\right)$ of $\lambda$ the partition of $m$ whose diagram obtained by reflection in the main diagonal. Hence $\lambda_{i}^{\prime}$ is the number of nodes in the ith column of $\lambda$. Define

$$
b(\lambda)=\sum_{i \geq 1}\left(\begin{array}{c}
\lambda_{i}^{\prime} \\
2
\end{array}\right) .
$$

If $\alpha=(i, j)$ is a cell of $\lambda$, then the corresponding hook is defined as $h_{\alpha}=$ $\lambda_{i}+\lambda_{j}^{\prime}-i-j+1$. 
Now we introduce the rational function in complex variables $u_{1}, \ldots, u_{m}$ with values in $H_{m}(r, s)$,

$\Psi\left(u_{1}, \ldots, u_{m}\right)=\prod_{k=1, \ldots, m-1}\left(T_{k}\left(u_{1}, u_{k+1}\right) T_{k-1}\left(u_{2}, u_{k+1}\right) \cdots T_{1}\left(u_{k}, u_{k+1}\right)\right) \cdot T_{w_{m}}^{-1}$ where the product is carried out in the order of $k=1, \cdots, m-1$. The following theorem is obtained by a similar argument as in the one-parameter case $[11]$.

Theorem 6.1. For the partition $\lambda$ of $m$ and a standard $\lambda$-tableau $T$, the idempotents $E_{T}^{\lambda}$ can be obtained by the consecutive evaluations

$$
E_{T}^{\lambda}=\left.\left.\left.f(\lambda) \Psi\left(u_{1}, \ldots, u_{m}\right)\right|_{u_{1}=\sigma_{1}}\right|_{u_{2}=\sigma_{2}} \cdots\right|_{u_{m}=\sigma_{m}}
$$

where

$$
f(\lambda)=\left(\frac{s}{r}\right)^{b\left(\lambda^{\prime}\right)} s^{-\left(\begin{array}{c}
m \\
2
\end{array}\right)\left(1-\frac{s}{r}\right)^{m}} \prod_{\alpha \in \lambda}\left(1-\left(\frac{s}{r}\right)^{h_{\alpha}}\right)^{-1} .
$$

Example 6.2. For $m=2$ and $\lambda=(2)$ we get

$$
E_{T}^{\lambda}=f(\lambda)\left(s T_{1}+r\right)
$$

where $f(\lambda)=\frac{1}{r+s}$, for the standard tableau $T=\underline{1 \mid 2}$, In particular, $\sigma_{1}=1$, $\sigma_{2}=\frac{s}{r}$.

Example 6.3. For $m=2$ and $\lambda=(1,1)$, we get

$$
E_{T}^{\lambda}=f(\lambda) \frac{s^{2}}{r}\left(1-T_{1}\right)
$$

where $f(\lambda)=\frac{r}{s(r+s)}$, for the standard tableau $T=\frac{1}{2}$, In particular, $\sigma_{1}=1$, $\sigma_{2}=\frac{r}{s}$.

Example 6.4. For $m=3, \lambda=(1,1,1), T=\frac{1}{\frac{1}{3}}$, we have $\left.\left.\left.\Psi\left(u_{1}, u_{2}, u_{3}\right)\right|_{u_{1}=\sigma_{1}}\right|_{u_{2}=\sigma_{2}}\right|_{u_{3}=\sigma_{3}}=\frac{s^{6}}{r^{3}}\left(1-T_{1}-T_{2}+T_{1} T_{2}+T_{2} T_{1}-T_{1} T_{2} T_{1}\right)$, 
and $f(\lambda)=\frac{r^{3}}{(s+r)\left(s^{2}+r s+r^{2}\right) s^{3}}$. Thus we get

$$
E_{T}^{\lambda}=\frac{s^{3}}{(s+r)\left(s^{2}+r s+r^{2}\right)}\left(1-T_{1}-T_{2}+T_{1} T_{2}+T_{2} T_{1}-T_{1} T_{2} T_{1}\right),
$$

which is the same as that in formula (5.7).

From Corollary 4.4, we have

$$
E_{T}^{\lambda}\left(V^{\otimes m}\right) \cong E_{T}^{\lambda}\left(\bigoplus_{\mu} V_{\mu} \otimes V^{\mu}\right)
$$

Since $E_{T}^{\lambda}$ annihilates the irreducible $H_{m}(r, s)$-module $V^{\lambda^{\prime}}$, we have

$$
E_{T}^{\lambda}\left(V^{\otimes m}\right) \cong E_{T}^{\lambda}\left(\bigoplus_{\mu} V_{\mu} \otimes V^{\mu}\right) \cong V_{\lambda} \otimes E_{T}^{\lambda} V^{\lambda}
$$

Furthermore, since $E_{T}^{\lambda}$ acts on the irreducible module $V^{\lambda}$ of $H_{m}(r, s)$ as a projector on a 1-dimensional subspace, we can get the following explicit description of the irreducible modules of $U_{r, s}\left(\mathfrak{s l}_{n}\right)$.

Theorem 6.5. For a partition $\lambda=\left(\lambda_{1}, \ldots, \lambda_{l}\right)$ of $m$ with length $l \leq n$ and $T$ a standard $\lambda$-tableau,

$$
V(\lambda)=E_{T}^{\lambda}\left(V^{\otimes m}\right)
$$

is the finite dimensional irreducible representation of $U_{r, s}\left(\mathfrak{s l}_{n}\right)$ with the highest weight $\sum_{i=1}^{n-1}\left(\lambda_{i}-\lambda_{i+1}\right) \bar{\omega}_{i}$.

Remark 6.6. We can see that Proposition 3.3 is a special case of Theorem 6.5. Actually, it is easy to check that $\check{R}\left(1, r^{-1} s\right)=s(s \check{R}+r)$. While from Example 6.2 for $\lambda=(2)$, we have

$$
E_{T}^{\lambda}=\frac{1}{r-s}\left(s T_{1}+r\right)
$$

Thus we have $S_{r, s}^{2}(V)=\check{R}\left(1, r^{-1} s\right) V^{\otimes 2}=E_{T}^{(2)}\left(V^{\otimes 2}\right)$. Similarly, using Example 6.3 we have $\Lambda_{r, s}^{2}(V)=\check{R}\left(1, r s^{-1}\right) V^{\otimes 2}=E_{T}^{(1,1)}\left(V^{\otimes 2}\right)$. 


\section{Acknowledgments}

We thank Alex Molev for discussions on fusion procedures. NJ gratefully acknowledges the partial support of Simons Foundation grant no. 523868, Humbolt Foundation, NSFC grant no. 11531004, "Mathematical Methods from Physics" in Summer of 2013 at KITPC, and MPI for Mathematics in the Sciences in Leipzig during this work. Liu thanks the support of NSFC grant no. 11701182 .

\section{References}

1. G. Benkart and S. Witherspoon, Two-parameter quantum groups and Drinfeld doubles, Algebr. Represent. Theory, 7 (2004), 261-286.

2. G. Benkart and S. Witherspoon, Representations of two-parameter quantum groups and Schur-Weyl duality, In: Hopf algebras, Lecture Notes in Pure and Appl. Math., 237, New York:Dekker, 2004, 62-92.

3. I. Cherednik, A new interpretation of Gelfand-Tzetlin bases, Duke Math. J., 54 (1987), 563-577.

4. R. Dipper and S. Donkin, Quantum GL ${ }_{n}$, Proc. London Math. Soc. (3), 63 (1991), 165-211.

5. R. Dipper and G. James, Blocks and idempotents of Hecke algebras of general linear groups, Proc. London Math. Soc., 54 (1987), 57-82.

6. V. Dobrev, Duality for the matrix quantum group $\mathrm{GL}_{p, q}(2, C)$, J. Math. Phys., 33 (1992), 3419-3430.

7. V. G. Drinfeld, Quantum groups, Proc. ICM, Vol. 1, 2 (Berkeley, Calif., 1986), 798-820, Amer. Math. Soc., Providence, RI, 1987.

8. J. Du, B. Parshall and J. Wang, Two-parameter quantum linear groups and the hyperbolic invariance of q-Schur algebras, J. London Math. Soc. (2), 44 (1991), 420-436.

9. M.-L. Ge, Y.-S. Wu and K. Xue, Explicit triogonometric Yang-Baxterization, Int. J. of Modern Phys. A 6(21) (1991), 3735-3779.

10. N. Hu, M. Rosso and H. Zhang, Two-parameter quantum affine algebra $U_{r, s}\left(\widehat{\mathfrak{s l}}_{n}\right)$, Drinfeld realization and quantum affine Lyndon basis, Commun. Math. Phys., 278 (2008), 453-486.

11. A. P. Isaev, A. I. Molev and A. F. Os'kin, On the idempotents of Hecke algebras, Lett. Math. Phys., 85 (2008), 79-90.

12. M. Jimbo, A q-difference analogue of $\mathrm{U}(\mathrm{g})$ and the Yang-Baxter equation, Lett. Math. Phys., 10 (1985), 63-69.

13. M. Jimbo, A q-analogue of $U\left(g l_{n}(N+1)\right)$, Hecke algebra, and the Yang-Baxter equation, Lett. Math. Phys., 11 (1986), 247-252. 
14. N. Jing, Quantum groups with two parameters, Contemp. Math., 134 (1992), 129-138.

15. N. Jing, K. Misra and M. Okado, $q$-Wedge modules for quantized enveloping algebras of classical type, J. Algebra, 230 (2000), 518-539.

16. N. Jing, L. Zhang and M. Liu, Wedge modules for two-parameter quantum groups, Contemp. Math., 602 (2013), 115-121.

17. Y. Manin, Topics in Noncommutative Geometry, Princeton Univ. Press, 1991.

18. M. Nazarov, A mixed hook-length formula for affine Hecke algebras, European J. Combin., 25 (2004), 1345-1376.

19. L. Poulain d'Andecy, Fusion procedure for the Yang-Baxter equation and Schur-Weyl duality, arXiv:1307.6808.

20. N. Reshetikhin, Multiparameter quantum groups and twisted quasitriangular Hopf algebras, Lett. Math. Phys., 20 (1990), 331-335.

21. M. Takeuchi, A two-parameter quantization of GL(n), Proc. Japan. Acad. Ser. A, 66 (1990), 112-114.

22. H. Weyl, The Classical Groups; Their Invariants and Representations, Princeton Univ. Press, Princeton, 1946. 